\title{
COMMENT
}

\section{Respiratory diseases in the era of COVID-19: Pearls and pitfalls}

\author{
MARIA BOLAKI ${ }^{1}$, ELIZA TSITOURA ${ }^{1}$, DEMETRIOS A. SPANDIDOS ${ }^{2}$, \\ EMMANOUIL K. SYMVOULAKIS ${ }^{3}$ and KATERINA M. ANTONIOU ${ }^{1}$ \\ ${ }^{1}$ Department of Respiratory Medicine, Laboratory of Molecular and Clinical Pneumonology, \\ Faculty of Medicine; ${ }^{2}$ Laboratory of Clinical Virology, Medical School; ${ }^{3}$ Clinic of Social and \\ Family Medicine, Faculty of Medicine, University of Crete, 71003 Heraklion, Greece
}

Received April 27, 2020; Accepted May 20, 2020

DOI: $10.3892 /$ etm.2020.8776

\begin{abstract}
Coronavirus disease 2019, a respiratory tract infection that has evolved into a pandemic, is expected to affect patients with underlying respiratory disease in a greater number and greater severity than patients with other underlying disorders. Whether this is true is an interesting question. However, the challenge both for the doctors and patients is to keep the respiratory disease in remission and prevent any exacerbations. Proper recommendations have been proposed for a wide range of respiratory disorders including chronic obstructive pulmonary disease (COPD), asthma and interstitial lung diseases, regarding the continuation or not of the treatment during this period and ways to maintain stability.
\end{abstract}

\section{Introduction}

Coronavirus disease 2019 (COVID-19) is an acute respiratory disease that can lead to respiratory failure and death. Patients with chronic respiratory diseases, such as chronic obstructive pulmonary disease (COPD) and asthma, would be anticipated to have an increased risk of infection and a more severe disease, something that seems not to be true in studies from China due to different reasons (1). Further research on the severity of comorbidities and all medication received by the patients is mandatory to shed light on these associations (1). Undoubtedly, the novel coronavirus (COVID-19) outbreak is a public health emergency (2). Defining the spectrum of clinical manifestations and the risk factors for severe COVID-19 infections is therefore an urgent research priority. A systematic

Correspondence to: Professor Katerina M. Antoniou, Department of Respiratory Medicine, Laboratory of Molecular and Clinical Pneumonology, Faculty of Medicine, University of Crete, 71003 Heraklion, Greece

E-mail: kantoniou@uoc.gr

Key words: COVID-19, SARS-CoV-2, respiratory disease, management review and meta-analysis was conducted to identify the symptoms and comorbidities predictive of COVID-19 severity (3). COPD was the most strongly predictive comorbidity for both severe disease [pooled odds ratio (pOR) 6.42, 95\% confidence interval (CI): 2.44-16.9] and intensive care unit (ICU) admission (pOR 17.8, 95\% CI: 6.56-48.2), rendering COPD patients particularly vulnerable to critical illness (3). Another meta-analysis aimed to explore the risk of severe COVID-19 in patients with pre-existing COPD and ongoing smoking history. The pOR of COPD and the development of severe COVID-19 was 4.38 while the OR of ongoing smoking was 1.98 (4).

\section{Chronic obstructive pulmonary disease}

Patients with COPD, a large proportion of patients with respiratory disease, should continue taking their regular inhaled and oral medicines in line with their individualized COPD self-management plan to ensure their COPD is as stable as possible. This includes those with COVID-19, or who are suspected of having it. Patients with COPD who are still smoking should be encouraged to stop to reduce the risk of poor outcomes from COVID-19 and their risk of acute exacerbations $(5,6)$. Similarly patients with asthma especially those with severe asthma should continue using inhaled corticosteroids because stopping can increase the risk of asthma exacerbation. There is no evidence that inhaled corticosteroids increase the risk of getting COVID-19 and this should be emphasized. In addition, they should continue biological treatment because there is no evidence that biological therapies for asthma suppress immunity (6-8).

\section{Interstitial lung diseases}

Patients with interstitial lung diseases (ILDs) represent a heterogeneous group of patients. Idiopathic pulmonary fibrosis patients already on anti-fibrotic drugs (pirfenidone and nintedanib) are not at any greater specific COVID-19 risk due to their treatment, therefore there is no need to alter drugs due to the outbreak. If an IPF patient on anti-fibrotic drugs is diagnosed with COVID-19 and admitted to hospital it is not considered harmful to pause the drugs for short 
periods of time (4-8 weeks). Other ILD patients already on immunosuppression should continue taking it unless they experience symptoms of infection. Starting immunosuppressive drugs in a non-rapidly progressing ILD patient during a viral pandemic raises concerns and if it is clinically safe delay should be considered of any initiation until pandemic is moving on. On the contrary, in rapidly progressive ILD cases e.g. vasculitis, connective tissue disease, ILD intravenous therapy should be discussed individually (9). It is important to consider that people with pulmonary fibrosis and their caregivers have a wide range of supportive care needs. They need more information on their disease and its treatment as well as better psychosocial and emotional support (10). Even patients with lymphangioleiomyomatosis (LAM) are strongly recommended not to stop taking sirolimus as a precautionary measure during the outbreak. Under certain circumstances, such as active infection with COVID-19 a dose reduction or interruption in sirolimus use may be necessary (LAM) (11).

\section{Rheumatic diseases}

Patients with rheumatic diseases consist of a special category because they receive immunosuppressive treatment and they usually present with lung involvement. Unfortunately, there is no knowledge on the infection rate and course of COVID-19 in rheumatologic conditions. Pending the data from national and international registries the general recommendation is that rheumatic disease patients taking immunosuppressive drugs should maintain their therapy, prevent infection by avoiding social contacts and discontinue immunosuppressants in case of infection (12). A special group of patients are those with systemic sclerosis (SSc) and ILD. These patients may be at risk for a more severe disease if they are infected with SARS-CoV-2 either due to underlying ILD and/or immunosuppressive treatment. It is postulated that they should continue immunosuppression to avoid SSc relapses. There is lack of evidence regarding discontinuation of immunosuppressive treatment in a SARS-CoV-2 positive patient. The degree of SSc-ILD and the risk of disease progression versus a higher risk of COVID-ILD evolution should be taken into account (13). A recently reported case of an SSc-ILD patient under treatment with tocilizumab suggests that temporary drug interruption may be advised (14).

\section{Cystic fibrosis}

Patients with cystic fibrosis represent a special group as well. Viral respiratory tract infections are more severe in patients with cystic fibrosis than in the general population, with an increased risk of complications and a negative impact on lung function. They have a phenotypic spectrum ranging from mild disease to chronic airways with respiratory compromise (15). Early data suggest that at present most patients with cystic fibrosis are doing an exceptional job avoiding SARS-CoV-2 infection, but they must remain dedicated to this task. Future research is expected to help us better understand factors that affect the severity of COVID-19 in people with cystic fibrosis (15). These patients should continue with all their usual self-care arrangements including airway clearance techniques, prophylactic medication and cystic fibrosis transmembrane conductance regulator (CFTR) therapies with some modifications (16).

\section{Lung cancer}

Lung cancer is a major challenge to clinicians because there is overlap between lung cancer evolution and COVID-19 infection from a radiological and clinical point of view. Obviously, these specific conditions require very different therapeutic approaches. The aggressiveness of lung malignancies does not allow for anticancer therapy to be withheld or postponed. Thus, the comprehensive management of patients with lung cancer during the COVID-19 pandemic is mandatory and should involve multidisciplinary lung cancer care teams (17). For patients with pneumonia, it is difficult to determine whether pneumonia has a COVID-19 viral cause or a bacterial cause (either primary or secondary to COVID-19). However, as COVID-19 becomes more prevalent in the community, patients presenting with pneumonia symptoms are more likely to have a COVID-19 viral pneumonia than a community-acquired bacterial pneumonia (18). Inadequate zinc status may predispose to infectious diseases of upper and lower respiratory tract and the antiviral effect of $\mathrm{Zn}$ against $\mathrm{nCoV} 2019$ should be further investigated (19).

Many questions on clinical management remain unanswered as these patients with underlying respiratory diseases may need ICU management. Undoubtedly, as COVID-19 spreads across the world, the ICU community must prepare for the challenges associated with this pandemic. It is crucial that ICU practitioners, governments, and policy makers prepare early for a substantial increase in critical care capacity (20).

\section{Acknowledgements}

Not applicable

\section{Funding}

No funding was received.

\section{Availability of data and materials}

The data and materials regarding the current study is available from the corresponding author on reasonable request.

\section{Authors' contributions}

All authors substantially contributed to the writing and revision of the manuscript, read and approved the final version.

\section{Ethics approval and consent to participate}

Not applicable.

\section{Patient consent for publication}

Not applicable. 


\section{Competing interests}

DAS is the Editor-in-Chief for the journal, but had no personal involvement in the reviewing process, or any influence in terms of adjudicating on the final decision, for this article. The other authors declare that they have no competing interests.

\section{References}

1. Halpin DMG, Faner R, Sibila O, Badia JR and Agusti A: Do chronic respiratory diseases or their treatment affect the risk of SARS-CoV-2 infection? Lancet Respir Med 8: 436-438, 2020.

2. World Health Organization (WHO): Report of the WHO-China Joint Mission on Coronavirus Disease 2019 (COVID-19). WHO, Geneva, 2020. https://www.who.int/publications-detail/ report-of-the-who-china-joint-mission-on-coronavirus-disease2019-(covid-19). Accessed February 28, 2020.

3. Jain V and Yuan JM: Systematic review and meta-analysis of predictive symptoms and comorbidities for severe COVID-19 infection. medRxiv: doi: https://doi.org/10.1101/2020.03.15.200 35360 .

4. Zhao Q, Meng M, Kumar R, Wu Y, Huang J, Lian N, Deng Y and Lin S: The impact of COPD and smoking history on the severity of Covid-19: A systemic review and meta-analysis. J Med Virol: April 15, 2020 (Epub ahead of print).

5. National Institute for Health and Care Excellence (NICE): COVID-19 rapid guideline: community-based care of patients with chronic obstructive pulmonary disease (COPD). NICE guideline [NG168). www.nice.org.uk/guidance/ng168. Accessed April 9, 2020.

6. Halpin DMG, Singh D and Hadfield RM: Inhaled corticosteroids and COVID-19: A systematic review and clinical perspective. Eur Respir J 55: 2001009, 2020.

7. National Institute for Health and Care Excellence (NICE): COVID-19 rapid guideline: severe asthma. NICE guideline [NG166). www.nice.org.uk/guidance/ng166. Accessed April 3, 2020 .

8. The Lancet Respiratory Medicine: Reflecting on World Asthma Day in the era of COVID-19. Lancet Respir Med 8: 423, 2020.

9. British Thoracic Society: British Thoracic Society Advice for Managing Interstitial Lung Disease Patients during COVID-19 pandemic. https://brit-thoracic.org.uk/media/455101/ bts-management-advice-for-ild-patients-v10-23-march-2020.pdf. Accessed March 23, 2020.

10. Lee JYT, Tikellis G, Corte TJ, Goh NS, Keir GJ, Spencer L, Sandford D, Khor YH, Glaspole I, Price J, et al: The supportive care needs of people living with pulmonary fibrosis and their caregivers: A systematic review. Eur Respir Rev 29: 190125, 2020 .
11. The LAM Foundation: The LAM Foundation COVID-19 Statement. https://thelamfoundation.org/Portals/0/Files/ Documents/COVID-19\%20Statement\%2003.18.20.pdf. Accessed March 18, 2020.

12. Ceribelli A, Motta F, De Santis M, Ansari AA, Ridgway WM, Gershwin ME and Selmi C: Recommendations for coronavirus infection in rheumatic diseases treated with biologic therapy. J Autoimmun 109: 102442, 2020.

13. Matucci-Cerinic M, Bruni C, Allanore $Y$, Clementi $M$, Dagna L, Damjanov NS, de Paulis A, Denton CP, Distler O, Fox D, et al: Systemic sclerosis and the COVID-19 pandemic: World Scleroderma Foundation preliminary advice for patient management. Ann Rheum Dis: April 29, 2020 (Epub ahead of print).

14. Mihai C, Dobrota R, Schröder M, Garaiman A, Jordan S, Becker MO, Maurer B and Distler O: COVID-19 in a patient with systemic sclerosis treated with tocilizumab for SSc-ILD. Ann Rheum Dis 79: 668-669, 2020.

15. ColomboC,BurgelPR,GartnerS, vanKoningsbruggen-RietschelS, Naehrlich L, Sermet-Gaudelus I and Southern KW: Impact of COVID-19 on people with cystic fibrosis. Lancet Respir Med 8: e35-e36, 2020.

16. National Institute for Health and Care Excellence (NICE): COVID-19 rapid guideline: cystic fibrosis. NICE guideline [NG170). www.nice.org.uk/guidance/ng170. Accessed April 9, 2020.

17. Calabrò L, Peters S, Soria JC, Di Giacomo AM, Barlesi F, Covre A, Altomonte M, Vegni V, Gridelli C, Reck M, et al: Challenges in lung cancer therapy during the COVID-19 pandemic. Lancet Respir Med: Apr 9, 2020 (Epub ahead of print).

18. National Institute for Health and Care Excellence (NICE): COVID-19 rapid guideline: managing suspected or confirmed pneumonia in adults in the community. NICE guideline [NG165). www.nice.org.uk/guidance/ng165. Accessed April 3, 2020.

19. Skalny AV, Rink L, Ajsuvakova OP, Aschner M, Gritsenko VA, Alekseenko SI, Svistunov AA, Petrakis D, Spandidos DA, Aaseth J, et al: Zinc and respiratory tract infections: Perspectives for COVID 19 (Review). Int J Mol Med 46: 17-26, 2020.

20. Phua J, Weng L, Ling L, Egi M, Lim CM, Divatia JV, Shrestha BR, Arabi YM, Ng J, Gomersall CD, et al; Asian Critical Care Clinical Trials Group: Intensive care management of coronavirus disease 2019 (COVID-19): Challenges and recommendations. Lancet Respir Med 8: 506-517, 2020.

This work is licensed under a Creative Commons Attribution-NonCommercial-NoDerivatives 4.0 International (CC BY-NC-ND 4.0) License. 